\title{
Sex determination and inbreeding depression in an ant with regular sib-mating
}

\author{
A Schrempf ${ }^{1}, \mathrm{~S}$ Aron $^{2}$ and J Heinze ${ }^{1}$ \\ ${ }^{1}$ Lehrstuhl Biologie I, University of Regensburg, Universitätsstraße 31, Regensburg D-93040, Germany; ${ }^{2}$ Behavioral and Evolutionary \\ Ecology, Université Libre de Bruxelles, Brussels, Belgium
}

\begin{abstract}
Haplodiploidy is one of the most widespread mechanisms of sex determination in animals. In many Hymenoptera, including all hitherto investigated social species, diploid individuals, which are heterozygous at the sex locus, develop as females, whereas haploid, hemizygous individuals develop as males (single-locus complementary sex determination, sl-CSD). Inbreeding leads to homozygosity at the sex locus, resulting in the production of diploid males, which are usually sterile and constitute a considerable fitness cost. Nevertheless, regular inbreeding without diploid male production is known from several solitary wasps, suggesting that in these species sex is not determined by sl-CSD but alternative mechanisms. Here, we examine sex determination in an ant with regular inbreeding, Cardiocondyla obscurior. The almost
\end{abstract}

complete absence of diploid males after 10 generations of brother-sister mating in the laboratory documents for the first time the absence of SI-CSD and CSD with two or a few unlinked sex loci in a species of social Hymenoptera. Queens, which mated with a brother, appeared to decrease the number of males in their brood, as expected from the relatedness relationships under inbreeding. In contrast, some colonies began to show signs of an inbreeding depression after several generations of sib-mating, such as shortened queen life span, higher brood mortality, and a shift to more male-biased sex ratios in some colonies, presumably due to lower insemination capability of sperm.

Heredity (2006) 97, 75-80. doi:10.1038/sj.hdy.6800846; published online 17 May 2006

Keywords: Cardiocondyla; haplodiploidy; sex determination; inbreeding depression; sl-CSD

\section{Introduction}

Arrhenotokous parthenogenesis (haplodiploidy), a system in which males develop from unfertilised eggs (and are haploid) and females develop from fertilised eggs (and are diploid), is one of the most widely distributed mechanisms of sex determination in animals (van Wilgenburg et al, 2006). In Hymenoptera, haplodiploidy appears to be commonly based on single-locus complementary sex determination (sl-CSD, Whiting, 1939, 1943). In sl-CSD, individuals heterozygous at the sex locus develop into females, whereas hemizygous, haploid individuals develop into males. As yet, sl-CSD has been documented in more than 60 species of Hymenoptera, including all studied social species (van Wilgenburg et al, 2006), and it has been suggested that sl-CSD is ancestral at least in the ichneumonoid and aculeate Hymenoptera (Butcher et al, 2000).

In species with sl-CSD, inbreeding leads to matched matings, in which eggs and sperm cells carry the same sex allele, and results in the production of diploid males, which are usually sterile or produce sterile, triploid progeny (Whiting, 1943; Stouthamer et al, 1992; Cook, 1993a; Krieger et al, 1999; but see Cowan and Stahlhut, 2004). The production of diploid males constitutes particularly high fitness costs in the social Hymenoptera, because queens mated with a male carrying a matching

Correspondence: A Schrempf, Lehrstuhl Biologie I, University of Regensburg, Universitätsstraße 31, Regensburg D-93040, Germany.

E-mail: alexandra.schrempf@biologie.uni-regensburg.de

Received 1 December 2005; accepted 11 April 2006; published online 17 May 2006 sex allele will produce 50\% diploid males instead of workers and are at a considerable disadvantage during colony founding (Ross and Fletcher, 1986). Species with sl-CSD, therefore, often show adaptations to ensure outbreeding, such as premating dispersal, the production of offspring of only a single sex, or asynchronous hatching of males and females (Cook and Crozier, 1995).

Nevertheless, regular sib-mating is common in a number of haplodiploids, including solitary parasitoid wasps, fig wasps, and a few socially parasitic ants. In these cases, it has been suggested that sex is determined by alternative mechanisms, such as multi-locus complementary sex determination (ml-CSD; Snell, 1935; Crozier, 1971) or genomic imprinting (Beukeboom, 1995; Naito et al, 2000). In ml-CSD, only individuals homozygous at all sex loci develop as diploid males. Thus, ml-CSD can be detected only through numerous generations of inbreeding, and diploid male load remains low as long as occasional outbreeding occurs (Crozier, 1971). CSD with two loci has been suggested for the sawfly Arge nigrinodosa (Naito et al, 2000), while both sl- and ml-CSD have been rejected in the solitary wasps Goniozus nephantidis (Cook, 1993a), Trichogramma spp. (Stouthamer and Kazmer, 1994), and Nasonia vitripennis (Skinner and Werren, 1980). In the latter case, it was suggested that sex is determined by genomic imprinting; in which case, loci could be differentially imprinted on paternal and maternal genes (Poire et al, 1993; Beukeboom, 1995).

Although previous research on sex determination in social ants, bees, and wasps has always given evidence for sl-CSD (van Wilgenburg et al, 2006), regular inbreeding occurs in Cardiocondyla, an ant genus characterized 
by local mating of young queens and males in their natal nests (Schrempf et al, 2005a). Based on microsatellite analyses it has been estimated that $83 \%$ of all young queens of Cardiocondyla batesii (Schrempf et al, 2005a), $72 \%$ of Cardiocondyla nigra (A Schrempf, unpublished), and $84 \%$ of Cardiocondyla obscurior (YY Du and A Schrempf, unpublished) mated with their brothers.

Taking advantage of the comparatively short generation time of C. obscurior, we investigated whether sex is determined by sl-CSD or CSD with a few loci. We performed inbreeding experiments over 10 generations and investigated the productivity and numerical sex ratio of colonies and the occurrence of diploid males. Our results indicate that sl-CSD and 2l-CSD is absent in this ant but at the same time reveal that prolonged inbreeding negatively affects queen longevity and male fecundity.

\section{Materials and methods}

\section{Study species}

C. obscurior Wheeler 1929 was collected in Bahia, Brazil, in September 2000 and kept in the laboratory at artificial temperature and light cycles $\left(12 \mathrm{~h} 30^{\circ} \mathrm{C} / 12 \mathrm{~h} 25^{\circ} \mathrm{C}\right)$. In addition to female sexuals and workers, colonies normally produce only wingless, fighting males (Heinze and Delabie, 2005). Under 'stressful' conditions, colonies also produce winged males (Cremer and Heinze, 2003). In our experiments, colonies produced both winged and wingless males, presumably because they were disturbed by the repeated removal of pupae and the frequent inspection of colony size.

\section{Inbreeding experiments}

We investigated the mechanism of sex determination by comparing the productivity and sex ratio in outbred and inbred experimental colonies, and by determining the ploidy of males. Diploid male production would result in a more male-biased sex ratio when diploid males survive and in a decreased production of workers and female sexuals when diploid males are culled during early development. In case of sl-CSD of half the fertilised eggs in $50 \%$ queens that mated with a brother are expected to be homozygous at the sex locus and to yield diploid males. In case of ml-CSD with two loci, $25 \%$ of the fertilised eggs in $25 \%$ of the inbred colonies should give diploid males. Multi-locus CSD with more than two loci similarly leads to increased male bias and decreased worker numbers, but more slowly than under sl-CSD and 2l-CSD.

In all, 10 'source' colonies with a single reproducing queen (mated with a male from an alien colony) were used to establish 16 inbred lines (from nine source colonies) and 12 control lines (from 10 source colonies) by transferring a female sexual pupa and a male pupa from the same or different source colonies into queenless groups consisting of 20 unrelated workers. To compare the productivity of experimental colonies, we standardized their size to 20 workers by removing all surplus workers. The number of worker pupae and eggs produced was recorded twice per week and all sexual pupae were removed and counted until the death of the queen (mean: 31 weeks). Cardiocondyla workers do not have ovaries (Heinze et al, 2006) and all eggs are therefore laid by the queen.

Sexual pupae from the first generation were inbred for nine additional generations over a period of three years ( $n=238 ; 10-37$ colonies per generation). In addition, we created 118 control colonies (six to 18 colonies per generation). Sexual pupae were removed and recorded twice per week as before, but colony size was no longer restricted to 20 workers and the number of worker pupae and eggs was only recorded at irregular intervals until queen death. The tenth generation was investigated only for a shift in sex ratio and not monitored until queen death. Colonies producing fewer than five sexual pupae were excluded from sex ratio analysis because they could have produced extreme ratios by chance. The proportion of excluded colonies did not differ between control (16 of 118) and inbred colonies (33 of $238 ; \chi^{2}=0.01 ; P=0.94$ ). All queens produced at least one worker, confirming that they were all inseminated.

The ploidy (haploid or diploid) of males and eggs from inbred colonies was determined by flow cytometry (Ploidy Analyser PAI, Partec; see Aron et al, 2003, 2005 for details). In addition, males and female sexuals from these colonies were mated with unrelated sexuals to determine whether male-bias and low productivity might be caused by decreased fecundity of inbred queens and decreased fertilization capability of inbred males. Eight winged and five wingless males from inbred colonies with a highly male-biased sex ratio and/or low productivity were dissected and examined for abnormalities and the amount of sperm in their seminal vesicles. To detect possible effects of inbreeding on the life span of queens, we compared the longevity of queens between inbred and outbred colonies. Queens, which did not survive the founding stage of 5 weeks or whose corpses were not found in the nest, were excluded from the analyses.

Normality of data was tested by Kolmogorov-Smirnovtests. Percentages, ratios, and non-normally distributed data were analysed by nonparametric statistics.

\section{Results}

In the first generation of brother-sister matings, 15 of 16 colonies produced enough offspring to allow the analysis of productivity and sex ratios. Inbred and control colonies did not differ in their productivity (total production of sexual pupae, control: $n_{\mathrm{C}}=9,23.0 \pm \mathrm{SD}$ 12.9; inbred: $n_{\mathrm{I}}=15,26.3 \pm 14.5$; Student's $t$-test, $t=\overline{0} .56$, $P=0.58$. Number of worker pupae per scan, control: $7.14 \pm 1.05$; inbred 7.17 $\pm 0.68 ; t=0.07, P=0.93$. Eggs per scan, control: $8.86 \pm 1.27$, inbred: $8.61 \pm 1.31 ; t=0.46$, $P=0.64$; Figure 1). By contrast, numerical sex ratios (proportion of females among sexuals) were considerably more female-biased in inbred than in control colonies (median and quartiles: $0.58 ; 0.53-0.68$ vs 0.35 ; 0.31-0.51, Mann-Whitney $U$-test, $U=14, P<0.005$; Figure 2). Sexual production was significantly associated with the life span of the mother queens (Spearman rank correlation: $\left.n=21, r_{\mathrm{S}}=0.56, P=0.008\right)$, but sex ratios were correlated neither with the life span of queens $\left(r_{\mathrm{S}}=0.02, P=0.92\right)$ nor with the total number of sexuals produced $\left(r_{\mathrm{S}}=0.17, P=0.45\right)$. All colonies produced both wingless and winged males, and the percentage of the 


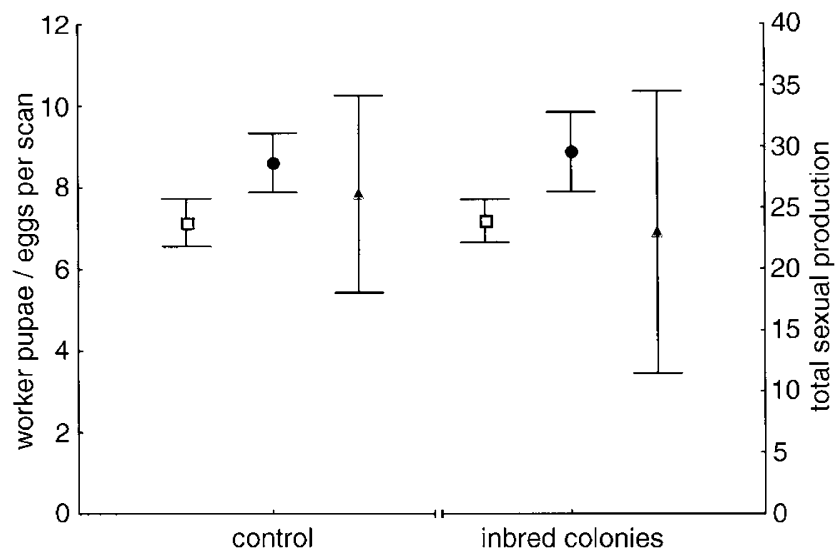

Figure 1 Productivity of inbred $(n=15)$ and control colonies $(n=9)$ of the ant $C$. obscurior. Mean $( \pm S D)$ number of worker pupae (white squares) and eggs (black circles) present during each scan (every three or four days) and the total number of sexuals (black triangles) produced during the whole lifetime of the queen are given.

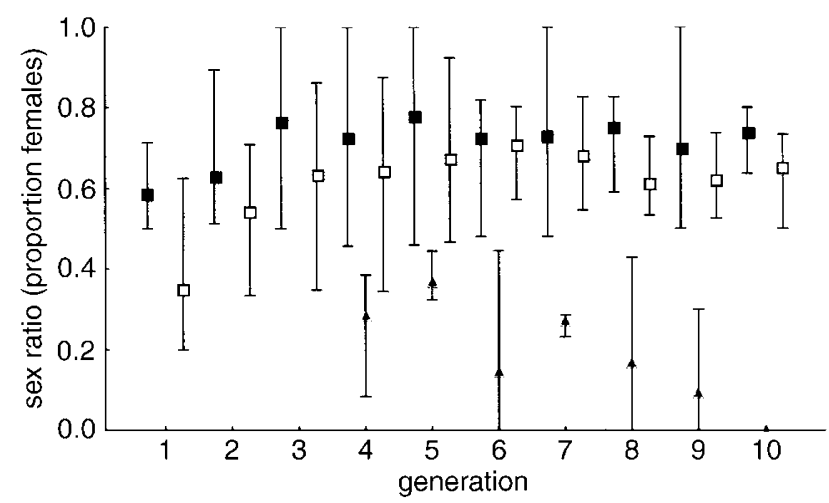

Figure 2 Sex ratio (female proportion, median, min-max) of $C$. obscurior control colonies (white squares; $n=108$ ), inbred colonies (black squares; $n=175$ ), and inbred colonies (black triangles) that produced extremely male-biased sex ratios and only few female offspring (black, $n=30$ ) over 10 generations.

latter did not differ between inbred and control colonies $(U=40.5, P=0.16)$.

Sex ratios were rather homogeneous in control colonies throughout subsequent generations (KruskalWallis-ANOVA, $n=99, H=10.48, P=0.23$; median: 0.65, quartiles: $0.55-0.71)$, but more female-biased than in the first generation $(U=85.5, P<0.001$; Figure 2$)$ as colony size was no longer restricted to 20 workers and colonies were not as frequently manipulated. Unstressed colonies produced a more female-biased sex ratio than stressed colonies (Cremer and Heinze, 2003). Sex ratios in inbred colonies did not differ among the ten generations ( $n=205, H=11.5, P=0.24)$, and the sex ratios of generations 2-10 did also not differ significantly from those in control colonies $\left(n_{\mathrm{C}}=99, n_{\mathrm{I}}=190, U=81633\right.$, $P=0.065$ ).

However, 12 inbred colonies, originating from three of the nine source colonies, had strongly male-biased sex ratios (median: 0.37, quartiles 0.22-0.43) in generations three to nine. During subsequent generations, daughter colonies derived from these colonies produced similarly

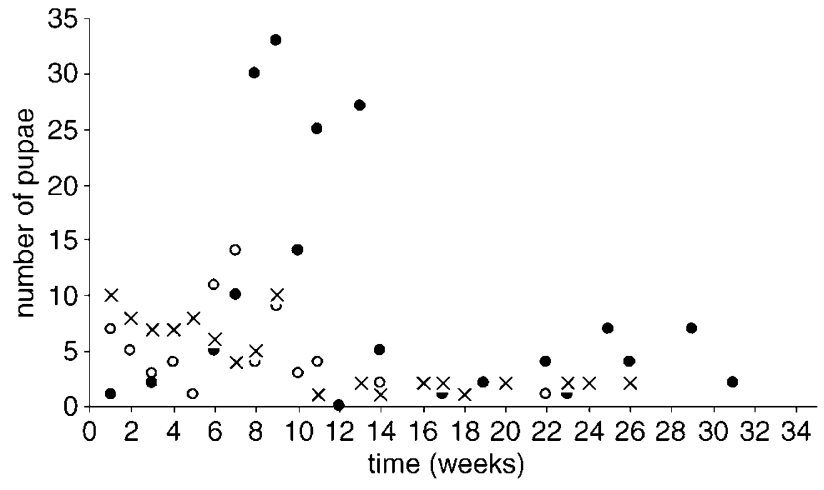

Figure 3 Production of males (black circles), female sexuals (white circles), and workers (crosses) per week in one inbred colony. The data suggest sperm depletion, because after 7 weeks, mainly males were produced.

or even more strongly male-biased sex ratios $(n=18$, median: 0.2, quartiles 0.0-0.29), until further crossing was no longer possible because of the lack of female sexuals. When the 30 colonies with extremely malebiased sex ratios are excluded, sex ratios were significantly more female-biased in inbred than in control colonies, like in the first generation $\left(n_{\mathrm{C}}=99, n_{\mathrm{I}}=160\right.$, $U=5050.50, P<0.001$; Figure 2).

Egg mortality in colonies with extreme male-bias was high (on average 50.7\%). Although queens produced large numbers of eggs (mean egg number/scan: $11.07 \pm 2.95)$, the number of larvae was low compared to other inbred colonies (median and quartiles: 4.02; 2.85-7.3 vs 20; 17-24; $U=12, P<0.001)$. Nevertheless, some of these colonies reared many sexuals, mostly males (up to 285) in addition to at least one worker. The percentage of males among the offspring of individual colonies increased with time, suggesting that queens ran out of sperm (Figure 3).

Flow cytometric analyses revealed that of 67 male pupae from nine colonies with extreme male bias only one (ie, 1.5\%) was diploid (winged male pupa from seventh inbred generation). The primary sex ratio (27 haploid eggs and 10 diploid eggs from five colonies with extreme male bias; sex ratio 0.27 ) was not significantly different from the secondary sex ratio (0.19), in particular when considering that diploid eggs also develop into workers. Thus, male bias does not result from the production of numerous, diploid males.

Nine males from inbred colonies with extreme malebias were allowed to mate with alien control queens. One male appeared to be infertile, as the queen laid only a few eggs and produced only a few males and no workers. Two queens produced numerous offspring with a slightly female-biased sex ratio (both 0.56). In five colonies, sex ratios were strongly male-biased (median: 0 , quartiles: 0-0.36), and in one colony only a few worker pupae and no sexuals were produced. Two daughter queens from inbred colonies with extreme male bias, which mated with unrelated control males, showed normal fertility and produced female-biased sex ratios (0.57 and 0.7$)$. The seminal vesicles of eight dissected males from inbred colonies with extreme male bias were filled with sperm and appeared to be normally developed. Seminal vesicles appeared to be less full than in control males and/or clumpy in three winged and one 


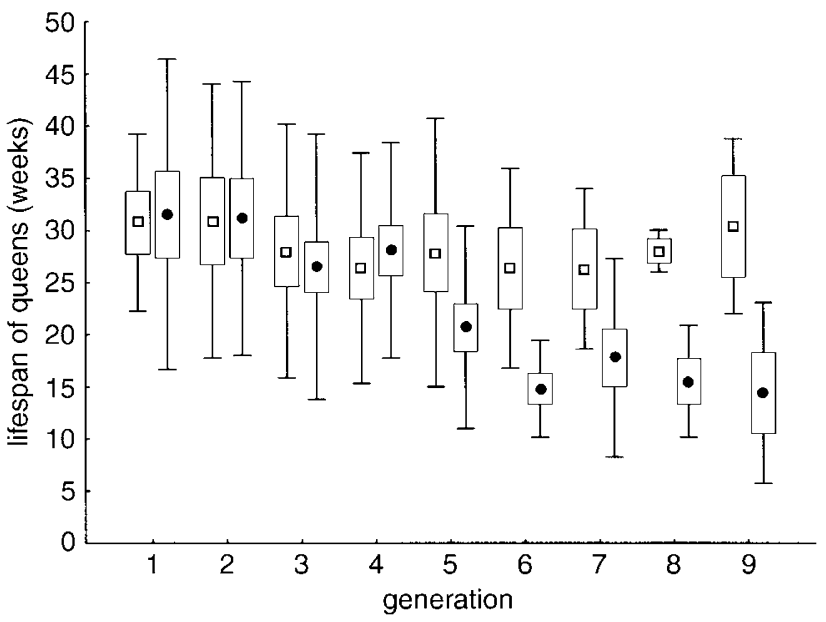

Figure 4 Mean life span $( \pm S D)$ of $C$. obscurior queens in control (white squares) and inbred (black circles) colonies over nine generations.

wingless male, and completely empty in another wingless male.

The mean life span did not differ between first generation queens mated with an unrelated male $(n=8$; $\mathrm{X} \pm \mathrm{SD}=30.8 \pm 8.48$ weeks) and queens mated with a brother $(n=13 ; 31.5 \pm 14.87$ weeks; Student's $t$-test, $t=0.12, P=0.90)$. Life span did also not differ between different generations of control colonies $(n=74$; ANOVA: $\mathrm{F}=0.37, P=0.93)$. In contrast, life span of queens from inbred colonies depended on the generation of inbreeding (Spearman-Rank-correlation: $n=124 ; r_{\mathrm{S}}=-0.466$, $P<0.001)$, that is, the mean life span of inbred queens was significantly reduced from the fifth generation on (Figure 4). In two colonies in which control females were mated with inbred males, queens died after 8 weeks.

\section{Discussion}

Our data document that sex in the ant C. obscurior is determined by a mechanism other than single locus complementary sex determination. Although alternative sex determination mechanisms have been invoked to explain regular inbreeding in a few socially parasitic ants (Crozier, 1971; Buschinger, 1989; Buschinger and Linksvayer, 2004), the absence of sl-CSD has not previously been proven in social Hymenoptera through controlled inbreeding experiments.

In contrast to expectations from sl-CSD, most inbred colonies were similar to control colonies in productivity and sex ratio. In the few inbred colonies with low productivity and male-biased sex ratios, flow cytometry revealed that only one of 67 mature males was diploid, and most of the eggs were haploid, too, so that we can reasonably exclude the production of large numbers of viable as well as of inviable diploid males. CSD with two loci is also unlikely in C. obscurior, as it would similarly result in a strong male bias or lowered brood production in most of the experimental colonies. Our data are compatible with ml-CSD with more than two loci. To clearly identify ml-CSD with several unlinked loci is difficult as both the proportion of inbred colonies that rear diploid males and the percentage of diploid males among their brood decrease drastically with the number of sex loci involved. According to Cook (1993a), ten generations of inbreeding are more than adequate to detect ml-CSD with 15 loci, provided that single motherson mating takes place. Ten generations of sib-mating should therefore be sufficient to reveal a system with a few loci. Furthermore, selection would be too weak to maintain heterozygosity at many loci, unless they had pleiotropic effects (Crozier, 1971; Bull, 1983), making ml-CSD unlikely. Genomic imprinting (Beukeboom, 1995), genic balance (Cunha and Kerr, 1957; Kerr, 1997), and the maternal effect model (Crozier, 1971; Cook, 1993a) are attractive alternative hypotheses for sex determination in C. obscurior, but cannot be tested without appropriate cytogenetic markers, such as the parental sex ratio chromosome in Nasonia (Beukeboom and Werren, 2000). Unfortunately, such markers are not available for ants.

The absence of sl-CSD protects queens that mate with close relatives from diploid male load and probably contributes to the success of Cardiocondyla as invasive tramp species (Heinze et al, 2006). Inbred queens of $C$. obscurior did not suffer decreased colony founding ability, productivity or longevity, and sex ratios were similar to those in control colonies. Nevertheless, prolonged inbreeding resulted in decreased queen life span and higher brood mortality. Our results indicate that haploid males contribute considerably to this inbreeding depression: the seminal vesicles of some males from inbred colonies contained less sperm and the male-biased sex ratios of some inbred colonies suggests sperm depletion or a lower fertilisation capability of sperm cells from inbred colonies. Although alternative explanations for male-biased sex ratios in inbred colonies are possible (eg, Wilkes, 1947; Hunter et al, 1993), our hypothesis of decreased sperm production or viability is corroborated by the observation that only two of nine female sexuals from control colonies that mated with males from inbred colonies managed to initiate a flourishing colony, while all others produced only a few larvae or male-biased sex ratios. In contrast, female sexuals from inbred colonies mated with control males had normal fertility and produced a female-biased sex ratio. Negative effects of inbreeding on the performance of haploid males have previously been documented in honeybees, where inbred males produce less sperm (Moritz, 1986), and the chalcidoid parasitoid Dahlbominus fuscipennis, where more inbred than outbred males are sterile (Wilkes, 1947). Obviously, inbreeding depression in haploid males cannot result from increased homozygosity at recessive, semi-lethal loci, but must be caused indirectly, for example, by maternal effects or epistatic interactions (Moritz, 1986).

Decreased longevity is common in inbred female Hymenoptera (Henter, 2003). In C. obscurior, queen life span was reduced after approximately five generations of inbreeding. The short life span of outbred C. obscurior queens, which mated with males from inbred colonies, suggests that low male quality may additionally affect queen longevity. Recent studies showed that mating increases queen life span, probably because seminal fluids affect the physiology of queens (Schrempf et al, $2005 \mathrm{~b}$ ). In natural populations of C. obscurior, the negative effects of prolonged inbreeding are probably prevented by occasional outbreeding, presumably through dispersing winged males. 
If the few inbred colonies with a highly male-biased sex ratio are disregarded, inbred colonies produced a significantly more female-biased sex ratio than control colonies. This can be explained either by an adaptive adjustment of sex ratios or by outbreeding depression. The latter may result from the disruption of co-adapted gene pools and result in similarly detrimental effects as inbreeding (Lynch, 1980; Peer and Taborsky, 2004). In C. obscurior, decreased egg fertilisation due to slight incompatibilities between egg and sperm might explain the comparatively more male-biased sex ratio in outbred colonies of C. obscurior. However, it would also lower productivity, for which there is no evidence in our colonies. Instead, the higher female bias in inbred colonies might stem from queens adaptively producing a more female-biased sex ratio when they mated with a related male, as inbreeding in Hymenoptera increases the relatedness between mothers and daughters but not between mothers and sons (Herre, 1985).

Our study adds a new facet to the steadily growing complexity of sex and caste differentiation mechanisms in social Hymenoptera, in particular ants. While until a few years ago, social Hymenoptera were mostly believed to be characterized by simple haplodiploid sex determination and environment-based caste differentiation, the last few years have revealed genetic caste determination, production of female sexuals by parthenogenesis, and production of males from fertilized eggs after the exclusion of the maternal nuclei (eg, Helms Cahan and Keller, 2003; Pearcy et al, 2004). The distribution of inbreeding and outbreeding in Cardiocondyla suggests that mechanisms of sex determination may be similarly plastic in social Hymenoptera as in solitary Hymenoptera, where they may vary within a single genus (Cook, 1993b; Beukeboom et al, 2000).

\section{Acknowledgements}

We thank B Lautenschläger, M Schiwek and T Wanke for their invaluable help in monitoring the colonies, JHC Delabie for providing support in Brazil, and $\mathrm{L}$ Beukeboom, $\mathrm{R}$ Crozier and two anonymous referees for helpful comments on the manuscript. Our research was funded by Deutsche Forschungsgemeinschaft (He $1623 / 20)$ and by grants from the Belgian Fonds National de la Recherche Scientifique (to SA). Collection and export of colonies was permitted by the Brazilian Minister of Science and Technology (RMX 004/02).

\section{References}

Aron S, de Menten L, Van Bockstaele DR (2003). Brood sex ratio determination by flow cytometry in ants. Mol Ecol Notes 3: 471-475.

Aron S, de Menten L, Van Bockstaele DR, Blank SM, Roisin Y (2005). When hymenopteran males re-invented diploidy. Curr Biol 15: 824-827.

Beukeboom L (1995). Sex determination in Hymenoptera - a need for genetic and molecular studies. Bioessays 17: 813-817.

Beukeboom L, Ellers J, Van Alphen JJM (2000). Absence of single-locus complementary sex determination in the braconid wasps Asobara tabida and Alysia manducator. Heredity 84: 29-36.

Beukeboom L, Werren JH (2000). The paternal-sex-ratio (PSR) chromosome in natural populations of Nasonia (Hymenoptera: Chalcidoidea). J Evol Biol 13: 967-975.
Bull JJ (1983). Evolution of Sex Determining Mechanisms. Benjamin/Cummings: Menlo Park, California.

Buschinger A (1989). Evolution, speciation, and inbreeding in the parasitic ant genus Epimyrma (Hymenoptera, Formicidae). J Evol Biol 2: 265-283.

Buschinger A, Linksvayer TA (2004). Novel blend of life history traits in an inquiline ant, Temnothorax minutissimus, with description of the male (Hymenoptera: Formicidae). Myrmecol Nachr 6: 67-76.

Butcher RDJ, Whitfield WGF, Hubbard SF (2000). Single-locus complementary sex determination in Diadegma chrysostictos (Gmelin) (Hymenoptera: Ichneumonidae). J Hered 9: 104-111.

Cook JM (1993a). Experimental tests of sex determination in Goniozus nephantidis (Hymenoptera, Bethylidae). Heredity 71: 130-137.

Cook JM (1993b). Sex determination in the Hymenoptera - a review of models and evidence. Heredity 71: 421-435.

Cook JM, Crozier RH (1995). Sex determination and population biology in the Hymenoptera. Trends Ecol Evol 10: 281-286.

Cowan DP, Stahlhut JK (2004). Functionally reproductive diploid and haploid males in an inbreeding hymenopteran with complementary sex determination. Proc Natl Acad Sci USA 101: 10374-10379.

Cremer S, Heinze J (2003). Stress grows wings: environmental induction of winged dispersal males in Cardiocondyla ants. Curr Biol 13: 219-223.

Crozier RH (1971). Heterozygosity and sex determination in haplodiploidy. Am Nat 105: 399-412.

Cunha AB, Kerr WE (1957). A genetical theory to explain sex-determination by arrhenotokous parthenogenesis. Forma Functio 1: 33-68.

Heinze J, Delabie JHC (2005). Population structure of the malepolymorphic ant Cardiocondyla obscurior. Stud Neotrop Fauna Environm 40: 187-190.

Heinze J, Cremer S, Eckl N, Schrempf A (2006). Stealthy invaders: the biology of Cardiocondyla tramp ants. Insect Soc 53: $1-7$.

Helms Cahan S, Keller L (2003). Complex hybrid origin of genetic caste determination in harvester ants. Nature 424: 306-309.

Henter HJ (2003). Inbreeding depression and haplodiploidy: experimental measures in a parasitoid and comparisons across diploid and haploid insect taxa. Evolution 57: 17931803.

Herre EA (1985). Sex ratio adjustment in fig wasps. Science 228: 896-898.

Hunter MS, Nur U, Werren JH (1993). Origin of males by genome loss in an autoparasitoid wasp. Heredity $\mathbf{7 0}$ 162-171.

Kerr WE (1997). Sex determination in honey bees (Apinae and Meliponinae) and its consequences. Braz J Genet 20 601-611.

Krieger MJB, Ross KG, Chang CW, Keller L (1999). Frequency and origin of triploidy in the fire ant Solenopsis invicta. Heredity 82: 142-150.

Lynch M (1980). The evolution of cladoceran life histories. $Q$ Rev Biol 55: 23-42.

Moritz RFA (1986). The origins of inbreeding depression in honeybees (Apis mellifera L.). BeeWorld 67: 157-163.

Naito T, Ishikawa M, Nishimoto Y (2000). Two-locus multipleallele sex determination in the rose sawfly Arge nigrinodosa. $3 r d$ Int Hym Congress, Canberra, Australia.

Pearcy M, Aron S, Doums C, Keller L (2004). Conditional use of sex and parthenogenesis for worker and queen production in ants. Science 306: 1780-1783.

Peer K, Taborsky M (2004). Outbreeding depression, but no inbreeding depression in haplodiploid ambrosia beetles with regular sibling mating. Evolution 59: 317-323.

Poire M, Periquet G, Beukeboom L (1993). The hymenopteran way of determining sex. Semin Dev Biol 3: 357-361. 
Ross KG, Fletcher DJC (1986). Diploid male production - a significant colony mortality factor in the fire ant, Solenopsis invicta (Hymenoptera: Formicidae). Behav Ecol Sociobiol 19: 283-291.

Schrempf A, Heinze J, Cremer S (2005b). Sexual cooperation: mating increases longevity in ant queens. Curr Biol 15: 267-270.

Schrempf A, Reber C, Tinaut A, Heinze J (2005a). Inbreeding and local mate competition in the ant Cardiocondyla batesii. Behav Ecol Sociobiol 57: 502-510.

Skinner SW, Werren JH (1980). The genetics of sex determination in Nasonia vitripennis. Genetics 94: s98.

Snell GD (1935). The determination of sex in Habrobracon. Proc Natl Acad Sci USA 21: 446-453.

Stouthamer R, Luck RF, Werren JH (1992). Genetics of sex determination and the improvement of biological control using parasitoids. Environ Entomol 21: 427-435.
Stouthamer R, Kazmer JD (1994). Cytogenetics of microbeassociated parthenogenesis and its consequences for gene flow in Trichogramma wasps. Heredity 73: 317-327.

Van Wilgenburg E, Driessen G, Beukeboom LW (2006). Single locus sex determination in Hymenoptera: an unintelligent design? Front Zool 3: 1-15.

Wheeler WM (1929). Ants collected by Professor F Silvestri in Formosa, the Malay Peninsula and the Philippines. Boll Lab Zool Gen Agr 24: 27-64.

Whiting PW (1939). Sex determination and reproductive economy in Habrobracon. Genetics 24: 110-111.

Whiting PW (1943). Multiple alleles in complementary sex determination of Habrobracon. Genetics 28: 365-382.

Wilkes A (1947). The effects of selective breeding on the laboratory propagation of insect parasites. Proc R Entomol Soc Lond B 134: 227-245. 